\title{
Induction of GDNF and GFRa-1 Following AAV1-Rheb(S16H) Administration in the Hippocampus in vivo
}

\author{
Dongyoung Yun ${ }^{1,2 \dagger}$, Min-Tae Jeon ${ }^{1,2 \dagger}$, Hyung-Jun Kim ${ }^{4 \dagger}$, Gyeong Joon Moon ${ }^{1,2}$, Shinrye Lee ${ }^{4}$, \\ Chang Man $\mathrm{Ha}^{5}$, Minsang Shin ${ }^{3,6}$ and Sang Ryong Kim ${ }^{1,2,3 *}$ \\ ${ }^{1}$ School of Life Sciences, ${ }^{2}$ BK21 plus KNU Creative BioResearch Group, ${ }^{3}$ Brain Science and Engineering Institute, Kyungpook \\ National University, Daegu 41566, ${ }^{4}$ Dementia Research Group and Neurodegenerative Disease Group, ${ }^{5}$ Research Division \\ and Brain Research Core Facilities, Korea Brain Research Institute, Daegu 41068, ${ }^{6}$ Department of Microbiology, School of \\ Medicine, Kyungpook National University, Daegu 41944, Korea
}

The activation of neurotrophic signaling pathways following the upregulation of glial cell line-derived neurotrophic factor (GDNF), a member of the transforming growth factor- $\beta$ family, has a potential neuroprotective effect in the adult brain. Herein, we report that hippocampal transduction of adeno-associated virus serotype 1 (AAV1) with a constitutively active form of ras homolog enriched in brain [Rheb(S16H)], which can stimulate the production of brain-derived neurotrophic factor (BDNF) in hippocampal neurons, induces the increases in expression of GDNF and GDNF family receptor $\alpha-1$ (GFR $\alpha-1)$, in neurons and astrocytes in the hippocampus of rat brain in vivo. Moreover, upregulation of GDNF and GFR $\alpha-1$ contributes to neuroprotection against thrombin-induced neurotoxicity in the hippocampus. These results suggest that AAV1-Rheb(S16H) transduction of hippocampal neurons, resulting in neurotrophic interactions between neurons and astrocytes, may be useful for neuroprotection in the adult hippocampus.

Key words: Rheb(S16H), Hippocampus, Astrocyte, GDNF, Neuroprotection

\section{INTRODUCTION}

Upregulation of glial cell line-derived neurotrophic factor (GDNF), a member of the transforming growth factor- $\beta$ family of trophic factors, can promote neuronal survival, axonal growth, and synapse formation in various neuronal cell populations $[1,2]$. In addition, the dysregulation of GDNF and GDNF family receptor $\alpha-1(G F R \alpha-1)$ contributes to neuronal death [3-5], and GDNF administration has neuroprotective effects against neurotoxininduced neurodegeneration $[1,6,7]$, suggesting that the GDNF/

Submitted November 18, 2019, Revised March 23, 2020 ,

Accepted March 24, 2020

* To whom correspondence should be addressed.

TEL: 82-53-950-7362, FAX: 82-53-943-2762

e-mail:srk75@knu.ac.kr

${ }^{\dagger}$ These authors contributed equally to this work.
GFRa-1 signaling pathway is a useful target for the development of anti-neurodegenerative therapies in the adult brain [8-10].

Many cellular effects of GDNF are initiated by binding to the specific receptor GFRa-1. One effect is that activation of the GDNF/GFRa-1 signaling pathway stimulates the intracellular Akt/mammalian target of rapamycin (mTOR) signaling pathway $[11,12]$. We recently reported that the transduction of adult dopaminergic neurons with adeno-associated virus serotype 1 (AAV1) with a constitutively active form of ras homolog enriched in brain $[\mathrm{Rheb}(\mathrm{S} 16 \mathrm{H})]$, which induces the sustained activation of mTOR complex 1 (mTORC1) by a resistance to the tuberous sclerosis $1 / 2$ (TSC1/2), protects the nigrostriatal dopaminergic system through the production of brain-derived neurotrophic factor (BDNF) and GDNF in an animal model of Parkinson's disease [10]. In the rat hippocampus, we also observed that induction of neuronal BDNF following AAV1-Rheb(S16H) administration contributes to the protection of hippocampal neurons from thrombin-induced 
neurotoxicity $[13,14]$. However, it is unclear whether Rheb(S16H) transduction of hippocampal neurons can promote the production of GDNF for neuroprotection in the hippocampus in vivo. In the present study, we investigate the following questions: whether AAV1-Rheb(S16H) administration upregulates GDNF expression in the hippocampus, whether the upregulation of GDNF is mediated by Rheb(S16H)-induced neuronal neurotrophic factors, and whether the activation of the GDNF/GFRa-1 signaling pathway following AAV1-Rheb(S16H) administration contributes to neuroprotection against thrombin-induced neurotoxicity in the rat hippocampus.

\section{MATERIALS AND METHODS}

\section{Production of AAV viral vectors}

All vectors used in these studies were the AAV 1 serotype as previously described $[10,14,15]$. A plasmid carrying Rheb was purchased from OriGene Technologies (Rockville, MD, USA). Rheb DNA was amplified and modified to incorporate a FLAG-encoding sequence at the 3'-end by expanded long-template PCR (Roche, Basel, Switzerland). Rheb(S16H) was generated with the Phusion Site-Directed Mutagenesis Kit (New England Biolabs, Ipswich, MA, USA) in the pGEM-T vector (Promega, Madison, WI, USA), and then cloned into an AAV packaging construct that utilizes the chicken $\beta$-actin promoter and contains a 3' WPRE (pBL). AAVs were produced by the University of North Carolina Vector Core, and the genomic titer of Rheb $(\mathrm{S} 16 \mathrm{H})$ was $2 \times 10^{12}$ viral genomes/ $\mathrm{ml}$. Enhanced green fluorescent protein (GFP), used as a control, was subcloned into the same viral backbone, and viral stocks were produced at titers of $1 \times 10^{12}$ viral genomes $/ \mathrm{ml}$.

\section{Institutional review of animal protocols}

Female Sprague Dawley (SD) rats (10-week-old, 220 240 g) were obtained from Daehan Biolink (Eumseong, Korea). All animal experiments were conducted in accordance with the Guidelines for Animal Care and Use of Kyungpook National University, approved by the Animal Care Committee at Kyungpook National University (No. KNU 2016-42).

\section{Intra-hippocampal injection}

SD Rats were anesthetized with $360 \mathrm{mg} / \mathrm{kg}$ chloral hydrate (Sigma, St. Louis, MO, USA) through intraperitoneal injection, and positioned in a stereotaxic apparatus (David Kopf Instrument, Tujunga, CA, USA). Two microliters of the viral vectors were injected into the right hippocampus of the SD rats (AP: $-3.8 \mathrm{~mm}$; ML: -2.4 $\mathrm{mm}$; DV: $3.0 \mathrm{~mm}$, relative to the bregma), according to the brain atlas [16]. Infusions of viral vectors were performed at a rate of 0.1 $\mu \mathrm{l} / \mathrm{min}$ over $20 \mathrm{~min}$ using 30 -gauge injection needles connected to a $10 \mu \mathrm{l}$ Hamilton syringe. The needle was maintained in position for an additional $5 \mathrm{~min}$ to diffuse the materials before being slowly retracted.

To examine the contribution of neurotrophic signal pathways for neuroprotection, we used neutralizing antibodies (NA) [10, 14]. Three weeks after virus injection, $200 \mathrm{ng}$ of NA alone or in combination with $20 \mathrm{U}$ of thrombin (Sigma) dissolved in $4 \mu$ l phosphatebuffered saline (PBS) were injected into the right hippocampal CA1 region as described above [13, 14]. The following NA were used: anti-BDNF (Santa Cruz Biotechnology, Dallas, TX, USA), anti-GDNF (R\&D Systems, Minneapolis, MN, USA), and antiGFRa-1 (Sigma).

\section{Rapamycin administration}

To determine the contribution of mTORC1 activity to GDNF induction, SD rats were intraperitoneally injected with the mTORC1 inhibitor, rapamycin (5 mg/kg dissolved in 4\% ethanol, 5\% Tween 80, and PEG400; LC Laboratory, Woburn, MA, USA) once a day for 3 weeks after AAV1-Rheb(S16H) injection until 4 weeks postinjection $[10,14]$.

\section{Immunohistochemical staining}

Animals were transcardially perfused, and then fixed brain sections (30 $\mu \mathrm{m}$ thick) were processed for immunohistochemical staining as previously described with some modifications [10, 14]. Briefly, brain sections were rinsed in PBS and then incubated at $4{ }^{\circ} \mathrm{C}$ with the primary antibody for $48 \mathrm{hr}$. After rinsing with PBS containing $0.5 \%$ bovine serum albumin, brain sections were incubated at room temperature with the appropriate biotinylated secondary antibody and processed with an avidin-biotin complex kit (Vector Laboratories, Burlingame, CA, USA). The signal was detected by incubating sections in $0.5 \mathrm{mg} / \mathrm{ml} \mathrm{3,3'-diaminobenzidine} \mathrm{(Sigma)}$ in $0.1 \mathrm{M}$ PBS containing $0.003 \% \mathrm{H}_{2} \mathrm{O}_{2}$. Stained samples were analyzed with bright-field microscopy (Axio Imager, Carl Zeiss, Germany). The primary antibodies were rabbit anti-BDNF (1:200, Santa Cruz Biotechnology), rabbit anti-p-4E-BP1 (1:1,000, Cell Signaling Technology, Danvers, MA, USA), goat anti-GDNF (1:400, R\&D Systems), rabbit anti-GFRa-1 (1:100, Sigma), and mouse anti-Neuronal nuclei (NeuN, 1:500; Millipore, Billerica, MA, USA). Brain sections were mounted on gelatin-coated slides, and then analyzed with bright-field microscopy (Axio Imager).

For immunofluorescence labeling, brain sections were rinsed and incubated for $48 \mathrm{hr}$ with one of the following conjugated pairs: mouse anti-NeuN (1:500, Millipore) and rabbit anti-FLAG (1:3,000, Sigma); mouse anti-GFAP (1:2,000, Millipore) and rabbit anti-FLAG (1:3,000, Sigma); mouse anti-GFAP (1:2,000, Milli- 
pore) and rabbit anti-p-4E-BP1 (1:1,000, Cell Signaling Technology); mouse anti-NeuN (1:500, Millipore) and goat anti-GDNF (1:200, R\&D Systems); mouse anti-GFAP (1:2,000, Millipore) and goat anti-GDNF (1:200, R\&D Systems); mouse anti-NeuN (1:500, Millipore) and rabbit anti-GFRa-1 (1:100, Sigma); or mouse antiGFAP (1:2,000, Millipore) and rabbit anti-GFRa-1 (1:100, Sigma). The sections were then rinsed and incubated with Texas Redconjugated anti-goat IgG, anti-mouse IgG or anti-rabbit IgG (1:400, Vector Laboratories), and fluorescein isothiocyanate-conjugated anti-rabbit IgG (1:400, Jackson Lab, West Grove, PA, USA) or antimouse IgG (1:400, Vector Laboratories) for $1 \mathrm{hr}$, and then washed and mounted with ProLong Gold antifade reagent (Invitrogen, Carlsbad, CA, USA). Stained sections were viewed using a fluorescence microscope (Axio Imager).

\section{Western blot analysis}

Western blot analysis was performed as described previously $[10,14]$. Briefly, tissues were homogenized and centrifuged at $4{ }^{\circ} \mathrm{C}$ for $15 \mathrm{~min}$ at $14,000 \times g$, the supernatant was transferred to a fresh tube, and the concentration was determined using a bicinchoninic acid kit (Bio-Rad Laboratories, Hercules, CA, USA). Samples were boiled at $100^{\circ} \mathrm{C}$ for 5 min before loading onto the gel, and equal amounts of protein $(30 \mu \mathrm{g})$ in loading buffer were loaded into each lane. Proteins separated by gel electrophoresis were transferred to polyvinylidene difluoride membranes (Millipore) using an electrophoretic transfer system (Bio-Rad Laboratories). Membranes were incubated overnight at $4{ }^{\circ} \mathrm{C}$ with the following specific primary antibodies: mouse anti- $\beta$-actin $(1: 1,000$, Santa Cruz Biotechnologies), goat anti-GDNF (1:1,000, R\&D Systems), rabbit antip-4E-BP1 (1:1,000, Cell Signaling Technology), rabbit anti-4EBP1 (1:1,000, Cell Signaling Technology), rabbit anti-BDNF (1:500, Santa Cruz Biotechnology), and rabbit anti-GFRa-1 (1:1,000, Sigma). After washing, the membranes were incubated with horseradish peroxidase-conjugated anti-rabbit $\operatorname{IgG}(1: 5,000$, Santa Cruz Biotechnologies), anti-mouse IgG or anti-goat IgG (1:5,000, Invitrogen) for $1 \mathrm{hr}$ at room temperature. Blots were developed with ECL western blotting detection reagents (GE Healthcare Life Sciences, Little Chalfont, UK). For semi-quantitative analyses, the density of the immunoblot bands was measured with a computer imaging device and accompanying software (Fuji Film, Tokyo, Japan).

\section{Primary astrocyte cultures}

Astrocyte cultures were prepared from brains by the method of McCarthy and de Vellis [17]. Briefly, whole brains were homogenized in a $70-\mu \mathrm{m}$ strainer. Cells were seeded in T75 culture flasks or $100 \mathrm{~mm}$ culture dishes. Cells were cultured at $37^{\circ} \mathrm{C}$ in a humidified atmosphere containing 5\% $\mathrm{CO}_{2}$. Culture media were changed initially after 5 days and every 2 days after that, and the cells were used after culture for 14 21 days. Secondary cultures of pure astrocytes were obtained by shaking mixed glial cultures at $250 \mathrm{rpm}$ for $4 \mathrm{hr}$, and then the culture medium was discarded. Astrocytes were dissociated using trypsin-EDTA (Life Technologies, Loughborough, UK) and centrifuged at 2,000 rpm for $30 \mathrm{~min}$. Astrocytes obtained were seeded onto plates and cultured in DMEM (Life Technologies) supplemented with 10\% heat-inactivated fetal bovine serum (FBS; Gibco, Loughborough, UK) and penicillinstreptomycin (Gibco). Animals used for cell cultures were acquired and cared for in accordance with the guidelines published in the National Institutes of Health Guide for the Care and Use of Laboratory Animals.

\section{Quantitative RT-PCR for GFRa-1 expression in astrocytes in vitro}

Cultured astrocytes were treated with recombinant human BDNF (100 ng/ml; Peprotech, Seoul, Korea) or GDNF (100 ng/ $\mathrm{ml}$; R\&D Systems) for $8 \mathrm{hr}$, and then RNA was extracted from cells with TRIzol reagent (Life Technologies). RNA isolation was performed using the RNeasy Mini Kit (Qiagen, Germantown, MD, USA) according to the manufacturer's instructions. cDNA synthesis was performed at $37^{\circ} \mathrm{C}$ for 120 min with $100 \mathrm{ng}$ of RNA using a High-Capacity cDNA Reverse Transcription kit (Applied Biosystems, Waltham, MA, USA). Quantitative RT-PCR was performed using the one-step SYBR PrimeScript RT-PCR kit (Perfect Real Time; Takara Bio Inc., Shiga, Japan) according to the manufacturer's instructions, followed by detection using an Applied Biosystems 7,500 Real-Time PCR system (Applied Biosystems). GAPDH was used as an internal control. The $2-\Delta \Delta \mathrm{Ct}$ method was used to calculate relative changes in gene expression, as determined by real-time PCR experiments.

\section{Primer sequences}

Mouse GFRa-1 Forward:5'-GGGCAGTCCCGT TCA TAT CA-3' Reverse: 5'-GGCTGCATCGAGGCA GTT-3'

Mouse GAPDH Forward: 5'-AAC TTT GGCATT GTG GAA GG-3' Reverse: 5'ACACATTGGGGGTAGGAACA-3’

\section{Counting of hippocampal CA1 neurons and efficiency of AAV1-Rheb(S16H) transduction}

CA1 neurons were counted as previously described [14]. Briefly, alternate sections were obtained at 3.3,3.6, 4.16, and $4.3 \mathrm{~mm}$ posterior to the bregma, and two hippocampal sections from each level (eight regions per animal) were used to count cells in each rectangular area $(1 \times 0.05 \mathrm{~mm})$ of CA1 regions. The number of cells was 
expressed quantitatively as a percentage in comparison with the contralateral control using a light microscope (Axio Imager) at a magnification of $200 \times$.

To measure the efficiency of AAV1-Rheb(S16H) transduction in the hippocampus in vivo, FLAG-colocalized neurons and nonneuronal cells such as astrocytes and microglia following double immunofluorescence labeling were counted in four rectangular boxes $(1 \times 0.3 \mathrm{~mm})$ in the hippocampus of each animal using a fluorescence microscope (Axio Imager). The ratio of FLAGcolocalized neurons and non-neuronal cells was expressed as a percentage relative to the total number of FLAG-colocalized cells.

\section{Statistical analysis}

All values are expressed as the mean \pm standard deviation. Multiple comparisons among groups were performed with the independent samples $t$-test or one-way analysis of variance followed by Tukey's post-hoc tests. All statistical analyses were performed using Sigma Stat software (Systat Software, San Jose, CA, USA).

\section{RESULTS}

\section{GDNF upregulation occurs in neurons and astrocytes in the AAV1-Rheb(S16H)-transduced rat hippocampus}

In the previous study [14], we reported that the transduction of Rheb(S16H) using AAV1 was well observed in neurons, but not in glia, and that its neuronal upregulation stimulated the production of neuronal BDNF via mTORC1 activation, contributing to neuroprotection in the hippocampus in vivo. Similar to our previous results [14], following an intra-hippocampal injection of AAV1Rheb(S16H), the hippocampal tissue shows apparent neuronal expression of the transduced proteins compared to glial expression in vivo (98.8\% vs. 1.2\% for neurons and astrocytes, respectively), as revealed by staining with anti-FLAG antibodies (Fig. 1B), and Rheb(S16H) upregulation induced an increase of BDNF in hippocampal neurons (Fig. 1C) at 3 weeks after viral injection (Fig. 1A). In addition, we observed that $\mathrm{Rheb}(\mathrm{S} 16 \mathrm{H})$ transduction significantly increased protein levels of BDNF compared to those in the contralateral hippocampus, as demonstrated by western blotting at 3 weeks after an intra-hippocampal injection (Fig. 1A and 1D; ${ }^{*} \mathrm{p}<0.05$ vs. contralateral controls). Although Rheb(S16H) expression was mainly observed in neurons in the Rheb $(\mathrm{S} 16 \mathrm{H})$-transduced hippocampus (Fig. 1B), the results of immunohistochemical staining at 3 weeks after viral injection (Fig. 1A) showed that the activation of $\mathrm{mTORC} 1$, as indicated by increases in phosphorylated 4E-BP1 (p-4E-BP1; Fig. 2A), and GDNF upregulation (Fig. $2 \mathrm{~B})$ were observed in both neurons and astrocytes in the AAV1$\mathrm{Rheb}(\mathrm{S} 16 \mathrm{H})$-injected rat hippocampus. Western blotting also showed that the levels of p-4E-BP1 and GDNF were significantly increased in the Rheb(S16H)-transduced hippocampus compared to the contralateral hippocampus (Fig. $2 \mathrm{C}$; ${ }^{*} \mathrm{p}<0.05$ vs. contralateral controls). There was no change in $\mathrm{p}-4 \mathrm{E}-\mathrm{BP} 1$ and GDNF levels in the hippocampus treated with AAV 1-GFP (Fig. 2C).

To examine whether the upregulation of GDNF is mediated by mTORC1 activation following Rheb(S16H) transduction, we measured the protein levels of $\mathrm{p}-4 \mathrm{E}-\mathrm{BP} 1$ and GDNF after intraperitoneal injections of rapamycin, a specific inhibitor for mTORC1 $[10,14]$, for 7 days (Fig. 1A). As demonstrated by western blotting, treatment with rapamycin significantly reduced the protein levels of p-4E-BP1 compared to those in intact controls and $\mathrm{Rheb}(\mathrm{S} 16 \mathrm{H})$-transduced rats that received no rapamycin [Fig. 2D; ${ }^{*} \mathrm{p}<0.05$ vs. intact controls and $\mathrm{Rheb}(\mathrm{S} 16 \mathrm{H})$ alone]. In addition, we observed that the upregulation of GDNF in the Rheb(S16H)treated hippocampus (Fig. 2C) was significantly attenuated by rapamycin treatment (Fig. 2D), indicating that the induction of GDNF was mediated by mTORC1 activation in the Rheb(S16H)transduced hippocampus.

\section{Upregulation of GFRa-1 expression in the Rheb(S16H)- transduced hippocampus}

GFR $\alpha$-1, a receptor specific for GDNF, is necessary for many cellular effects of GDNF in the brain, and the activation of the GDNF/GFRa-1 signaling pathway is important for neuronal survival and proper function in various neuronal cell populations $[1,2$, 12]. In the Rheb(S16H)-transduced hippocampus, we additionally observed that the protein levels of GFRa-1 were increased both in hippocampal neurons and astrocytes, as demonstrated by immunohistochemical staining and western blotting at 3 weeks after viral injection (Fig. 3A, 3B, and 3C; ${ }^{*} \mathrm{p}<0.01$ vs. controls). Moreover, the increases in GFRa-1 expression by Rheb(S16H) transduction were significantly inhibited by treatment with NA against BDNF or GDNF, respectively, as demonstrated by western blotting at 1 day after NA treatment [Fig. 1A and $4 \mathrm{~A} ;{ }^{*} \mathrm{p}<0.05$ vs. Rheb $(\mathrm{S} 16 \mathrm{H})$ alone], suggesting that the upregulation of GFR $\alpha-1$ is mediated by increases in neurotrophic factors such as BDNF or GDNF following Rheb(S16H) transduction.

Other reports have shown that GDNF and BDNF each could play a role as a potent inducer for GFRa-1 expression in neurons $[18,19]$. However, it is unclear whether the upregulation of GDNF and BDNF can induce an increase in GFRa-1 expression in astrocytes. To determine whether these factors induced by $\mathrm{Rheb}(\mathrm{S} 16 \mathrm{H})$ transduction contribute to the upregulation of GFRa-1 in astrocytes in vivo (Fig. 3), we investigated GFRa-1 expression in the Rheb(S16H)-transduced hippocampus with or without NA against BDNF or GDNF, and measured gene levels of 

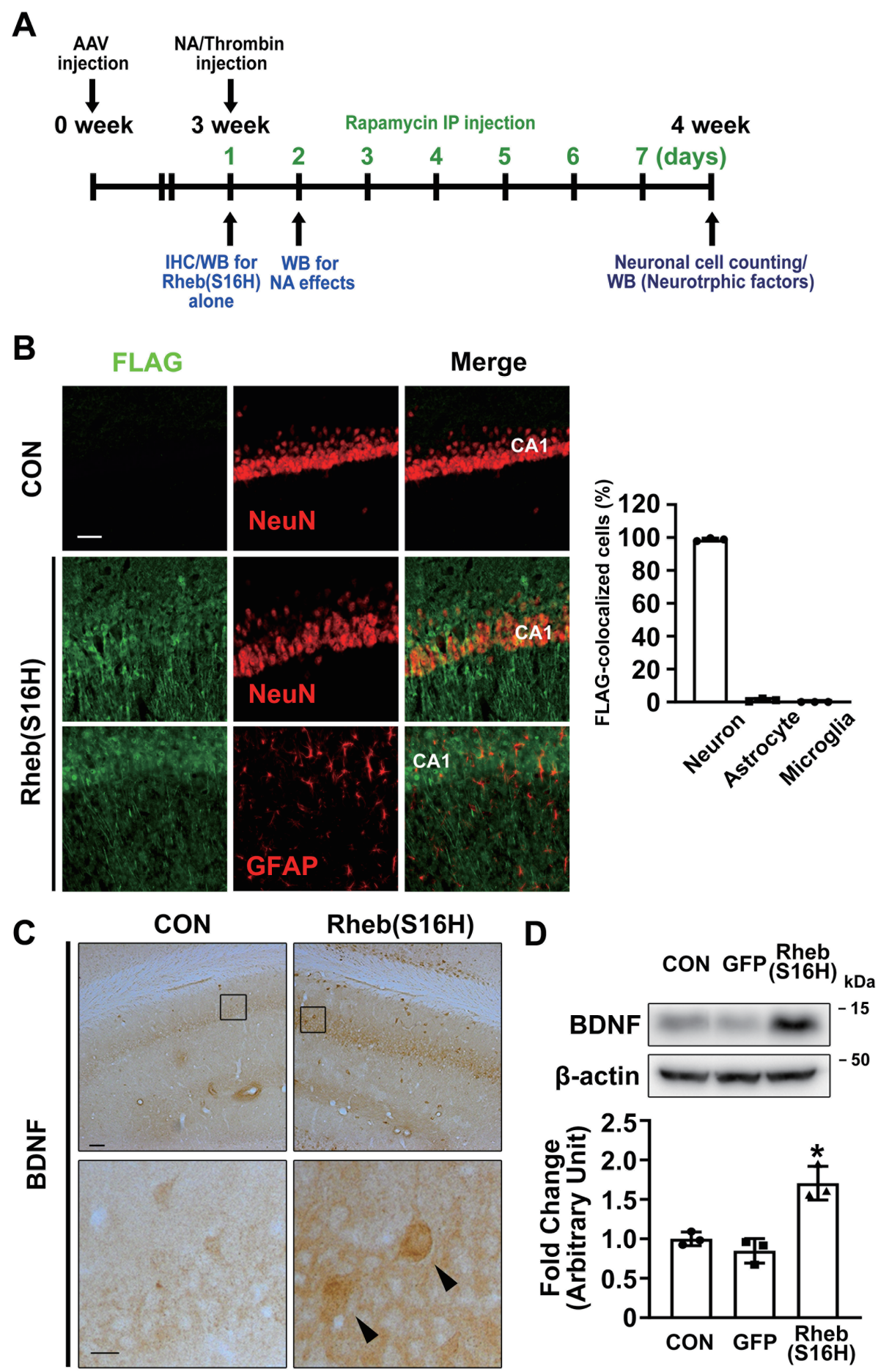

Fig. 1. Experimental schematic and transduction of hippocampal neurons with AAV1-Rheb(S16H) in SD rats. (A) Experimental schematic for investigating the effects of AAV1-Rheb(S16H) administration in the rat hippocampus in vivo. (B) Double immunofluorescence staining to visualize coexpression patterns of FLAG and NeuN, or FLAG and GFAP in the Rheb(S16H)-transduced hippocampus. Scale bar, $50 \mu \mathrm{m}$. The graph represents the ratio of FLAG-colocalized cells between neurons and non-neuronal cells in the AAV1-Rheb(S16H)-injectied hippocampus (n=3 per group). (C) Immunohistochemical staining of BDNF expression following to Rheb $(\mathrm{S} 16 \mathrm{H})$ transduction of hippocampal neurons in vivo. Lower images are magnified photomicrographs of the insets. Scale bars: upper images, $100 \mu \mathrm{m}$; lower images (insets), $20 \mu \mathrm{m}$. (D) Western blot analysis for BDNF expression in the controls, AAV1-GFP- and AAV1-Rheb(S16H)-injected hippocampus of rat brains. Differences among groups were evaluated by one-way analysis of variance and Tukey's post-hoc test $\left({ }^{*} \mathrm{p}<0.05\right.$ vs. $\mathrm{CON} ; \mathrm{n}=3$ per group). 
A

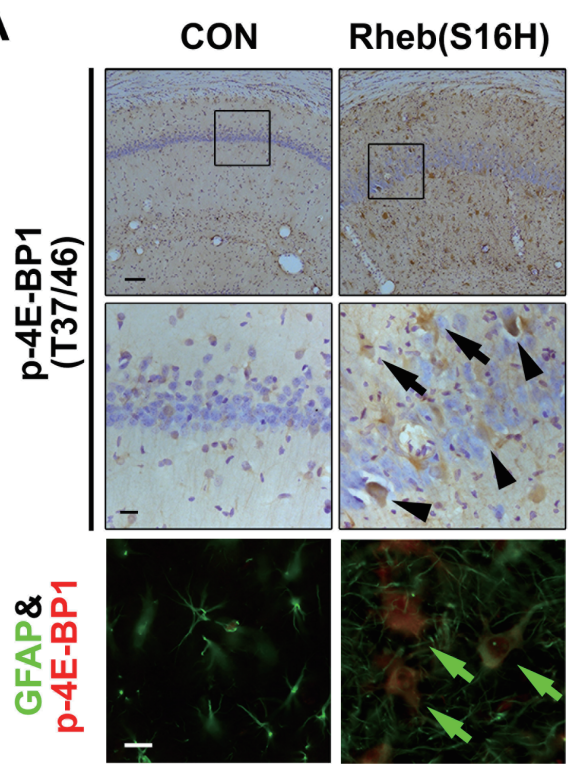

C
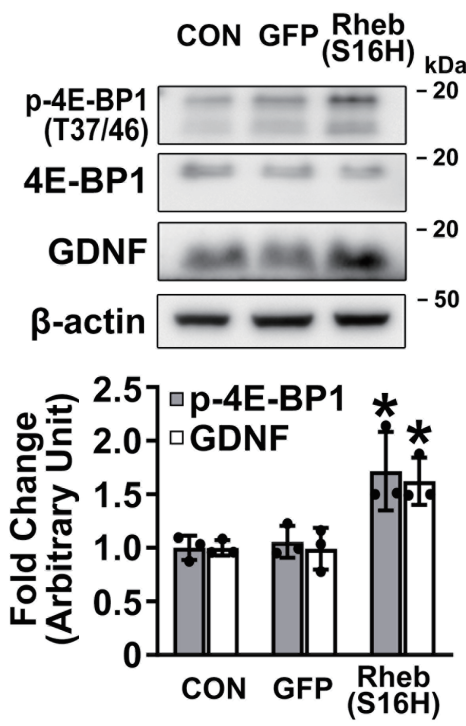

B
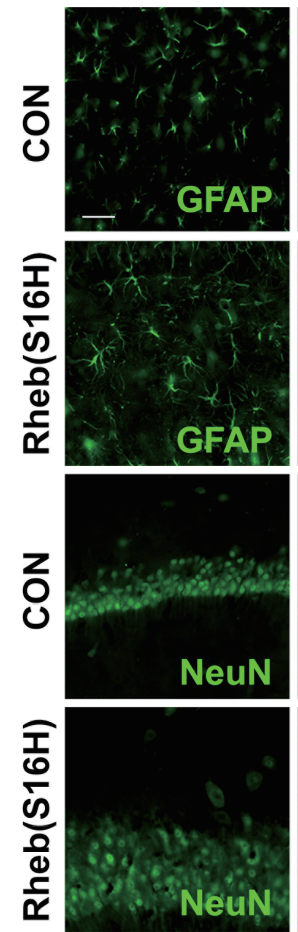

GDNF
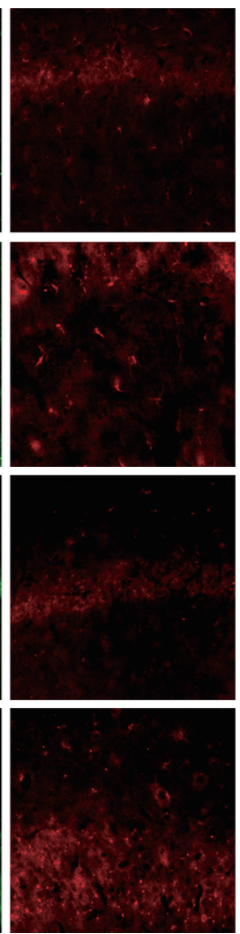

Merge
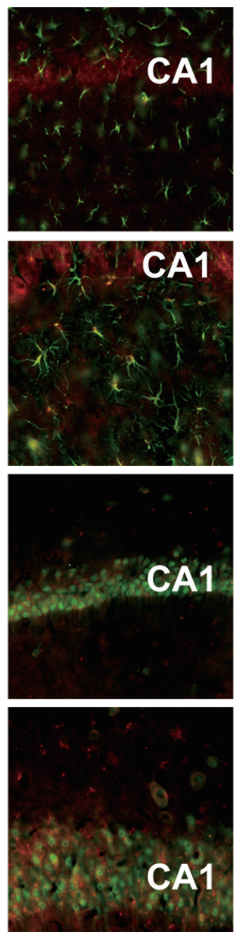

D
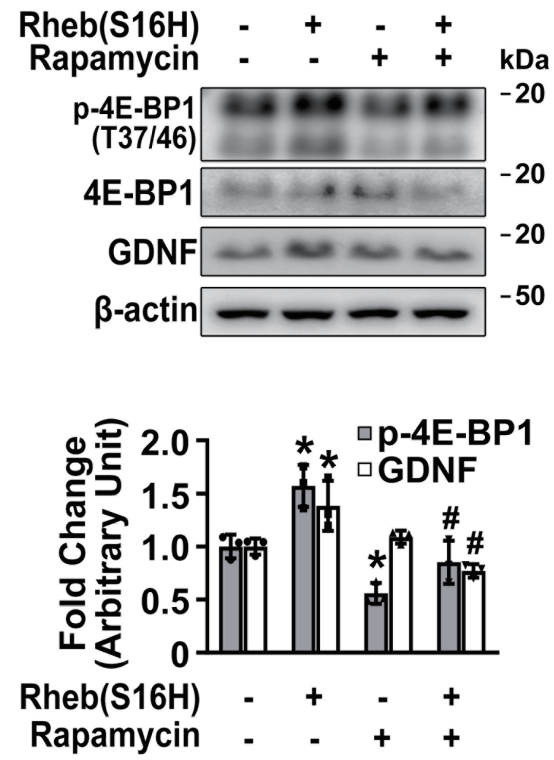

Fig. 2. mTORC1 activation and GDNF upregulation in the Rheb(S16H)-transduced hippocampus. (A) Immunohistochemical staining results of p-4E$\mathrm{BP} 1$, indicating $\mathrm{mTORC} 1$ activation, showed increases in p-4E-BP1 expression in neurons (arrow heads) and non-neuronal cells (arrows), as demonstrated in the magnified photomicrographs of the rectangular insets in the CA1 layer in the Rheb(S16H)-transduced hippocampus. Scale bars: upper images, $100 \mu \mathrm{m}$; center images (insets), $20 \mu \mathrm{m}$. Astrocytic mTORC1 activation in the Rheb(S16H)-expressed hippocampus was confirmed by double immunofluorescence staining for GFAP and p-4E-BP1 (green arrows). Scale bar: lower images, $20 \mu \mathrm{m}$. (B) Double immunofluorescence staining to visualize co-expression patterns of GDNF and NeuN, or GDNF and GFAP in the Rheb(S16H)-transduced hippocampus. Scale bar, $50 \mu \mathrm{m}$. (C) Western blot analysis for $\mathrm{p}-4 \mathrm{E}-\mathrm{BP} 1,4 \mathrm{E}-\mathrm{BP} 1$, and GDNF expression in the controls, AAV1-GFP- and AAV1-Rheb(S16H)-injected hippocampus of rat brains. Differences among groups were evaluated by one-way analysis of variance and Tukey's post-hoc test $\left({ }^{*} \mathrm{p}<0.05 \mathrm{vs}\right.$. CON; $\mathrm{n}=3$ per group). (D) Western blot analysis for mTORC1 activity (p-4E-BP1 and 4E-BP1) and GDNF expression in the hippocampus of rats treated with rapamycin (5 mg/kg) and AAV1$\mathrm{Rheb}(\mathrm{S} 16 \mathrm{H})$ compared to AAV1-Rheb(S16H) alone. Differences among groups were evaluated by one-way analysis of variance and Tukey's post-hoc analysis [ ${ }^{*} \mathrm{p}<0.05$ vs. CON, $\# \mathrm{p}<0.01$ vs. Rheb( $\left.\mathrm{S} 16 \mathrm{H}\right)$ alone; $\mathrm{n}=3$ per group]. 
A
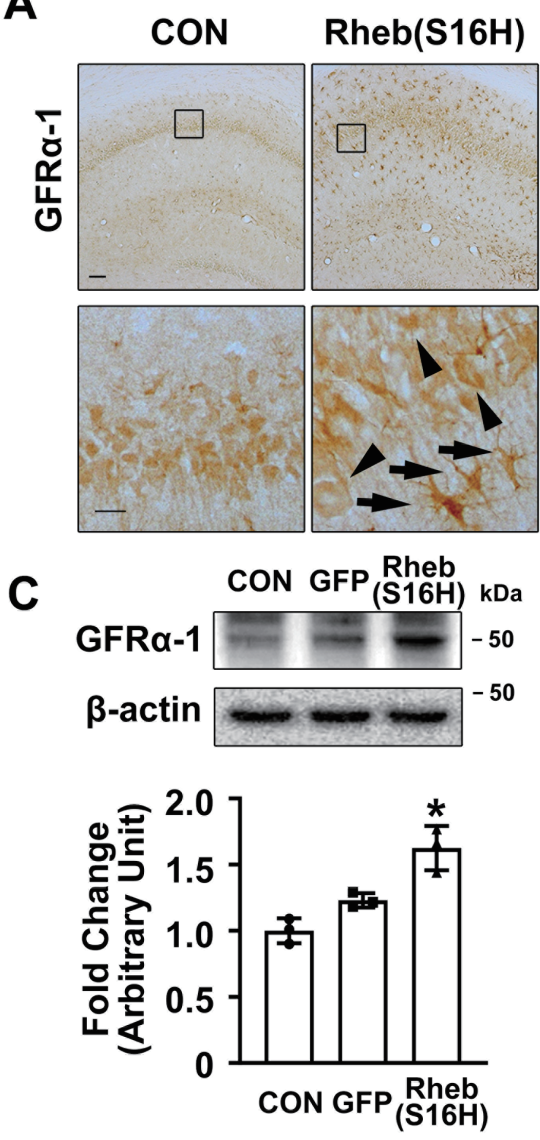

B

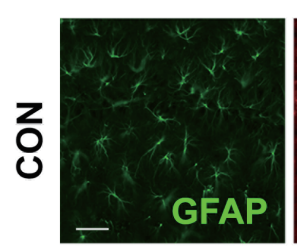

GFRa-1
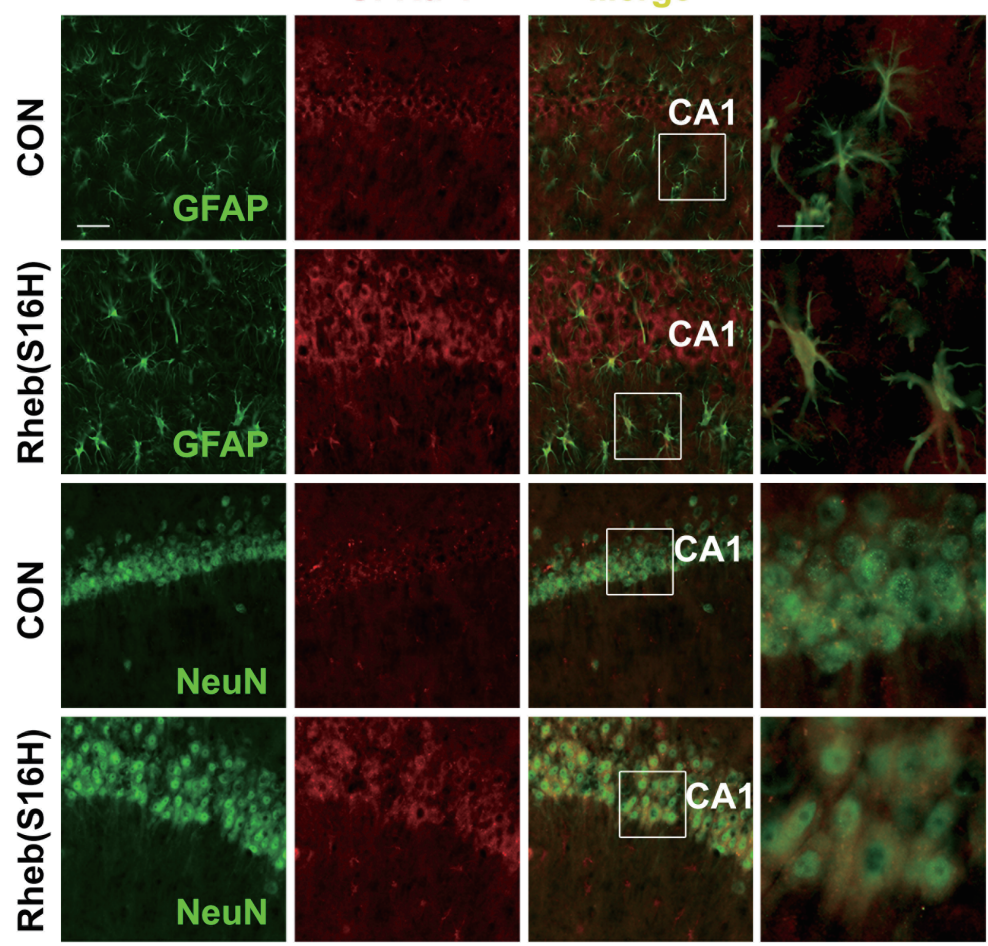

Fig. 3. Increases in GFRa-1 expression in the AAV1-Rheb(S16H)-treated hippocampus. (A) Representative images of immunostaining for GFRa-1. Immunohistochemical staining results showed the increases in GFRa-1 expression both in neurons (arrow heads) and non-neuronal cells (arrows) in the Rheb(S16H)-expressing hippocampus, as demonstrated by the magnified photomicrographs of the insets in the CA1 layer. Scale bars: upper images, $100 \mu \mathrm{m}$; lower images (insets), $20 \mu \mathrm{m}$. (B) Double immunofluorescence staining to visualize co-expression patterns of GFRa-1 and NeuN, or GFR -1 and GFAP in the Rheb(S16H)-transduced hippocampus. Scale bars: images in first three columns from left, $50 \mu \mathrm{m}$; images in far-right column (insets), $20 \mu \mathrm{m}$. (C) Western blot analysis for GFRa-1 expression in the controls, AAV1-GFP- and AAV1-Rheb(S16H)-injected hippocampus of rat brains. Differences among groups were evaluated by one-way analysis of variance and Tukey's post-hoc analysis; $\left({ }^{*} \mathrm{p}<0.01 \mathrm{vs}\right.$. CON; $\mathrm{n}=3 \mathrm{per}$ group).

GFR $\alpha-1$ in primary astrocyte cultures treated with $100 \mathrm{ng} / \mathrm{ml} \mathrm{re}$ combinant BDNF or GDNF. Astrocytic GFR $\alpha$ - 1 upregulation was attenuated by treatment with GDNF NA, but not BDNF NA, in the Rheb(S16H)-transduced hippocampus (Fig. 4B). Consistently, the results obtained by quantitative RT-PCR for GFRa-1 showed that GDNF treatment significantly increased the levels of GFRa1 mRNA in astrocytes compared to that in cells receiving vehicle only (Fig. 4C; ${ }^{*} \mathrm{p}<0.001$ vs. controls), but not in BDNF-treated astrocytes (Fig. 4D). However, treatment with NA against BDNF significantly attenuated the upregulation of astrocytic GDNF levels seen in the Rheb $(\mathrm{S} 16 \mathrm{H})$-transduced hippocampus compared to the levels in Rheb(S16H) alone [Fig. 4A; ${ }^{*} \mathrm{p}<0.05$ vs. Rheb $(\mathrm{S} 16 \mathrm{H})$ alone]. These results suggest that the increase in astrocytic GFR $\alpha-1$ following Rheb(S16H) transduction in the hippocampus in vivo is mediated by GDNF upregulation, and that BDNF may indirectly contribute to the induction of GFRa-1, even though there was no increase in the expression of GFRa-1 in the BDNF-treated astrocyte cultures.

\section{Neuroprotection by the activation of the GDNF/GFRa-1 sig- naling pathway in the Rheb(S16H)-transduced hippocam- pus in vivo}

To examine neuroprotective effects of upregulated GDNF and GFRa-1 expression in the hippocampus (Fig. 2 and 3), we evaluated the effects of treatment with NA against GDNF and GFRa1 , respectively, in the Rheb(S16H)-transduced hippocampus. Rats received a unilateral injection of thrombin, which can act as a neurotoxin in the hippocampus [13, 14], at 3 weeks after viral injection, and then the hippocampal sections were immunohistochemically stained with anti-NeuN at 1 week after thrombin 
A
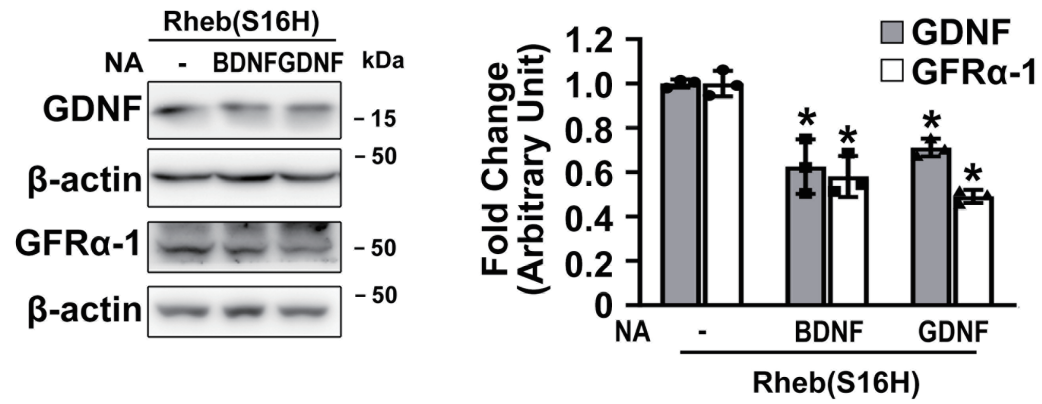

B

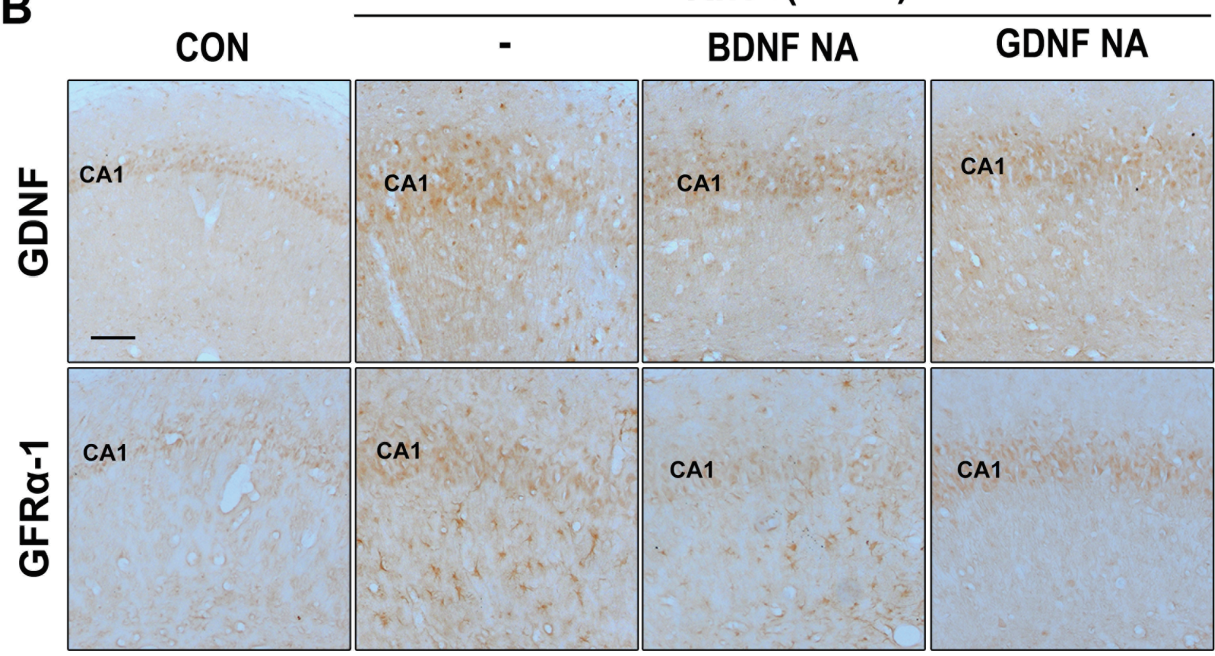

C

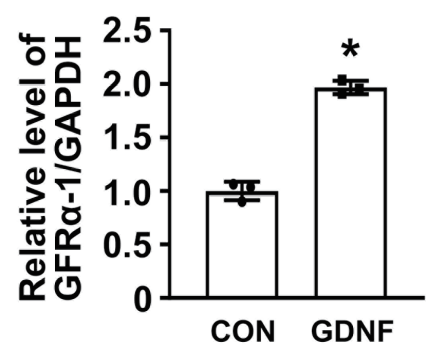

D

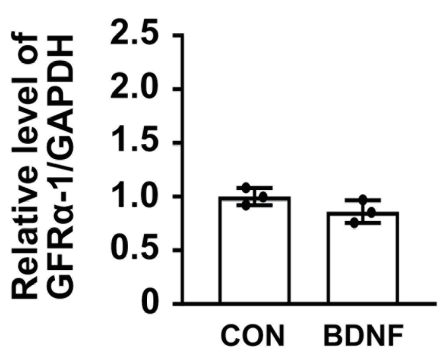

Fig. 4. Astrocytic GDNF and GFRa-1 expression following increases in neurotrophic factors in vivo and in vitro. (A, B) Western blot (A) and immunohistochemical (B) analysis for GDNF and GFRa-1 expression in the hippocampus of AAV1-Rheb(S16H)-injected rat brains, with or without neutralizing antibodies (NA; $200 \mathrm{ng}$ ) against BDNF or GDNF. Differences among groups were evaluated by one-way analysis of variance and Tukey's posthoc analysis [ ${ }^{*} \mathrm{p}<0.05 \mathrm{vs}$. Rheb(S16H) alone; $\mathrm{n}=3$ per group]. Scale bar, $100 \mu \mathrm{m}$. (C, D) Quantitative RT-PCR analyses for GFR $\alpha-1$ in primary astrocyte cultures showed significant increases in GFR $\alpha-1$ expression in the GDNF (100 ng/ml; C)-treated astrocyte cultures compared to vehicle-treated controls $(\mathrm{CON})$, but no change in response to treatment with $\operatorname{BDNF}(100 \mathrm{ng} / \mathrm{ml} ; \mathrm{D})$. Differences among groups were evaluated by $t$-test analysis $\left({ }^{*} \mathrm{p}<0.001 \mathrm{v} s\right.$. $\mathrm{CON} ; \mathrm{n}=3$ per group).

treatment or co-treatment with thrombin and NA (Fig. 1A). Immunohistochemical staining showed that Rheb(S16H) transduction of hippocampal neurons significantly preserved the number of NeuN-positive neurons from thrombin-induced neurotoxicity in the hippocampal CA1 regions by $92 \%$ compared to that of the contralateral controls (Fig. 5 ; $\# \mathrm{p}<0.001$ vs. thrombin alone). However, the inhibition of GDNF, GFRa-1, and BDNF signaling with those specific NA significantly attenuated the neuroprotective effects of Rheb(S16H) transduction by $23 \%, 31 \%$ and $24 \%$, respectively, compared to Rheb( $\mathrm{S} 6 \mathrm{H})$ and thrombin treated group [Fig. $5 ;{ }^{\$} \mathrm{p}<0.05$ vs. Rheb(S16H) and thrombin]. These results show that the activation of GDNF/GFR $\alpha-1$ signaling following Rheb(S16H) transduction contributes to the protection of hippocampal neurons from thrombin-induced neurotoxicity in the hippocampus in vivo. 

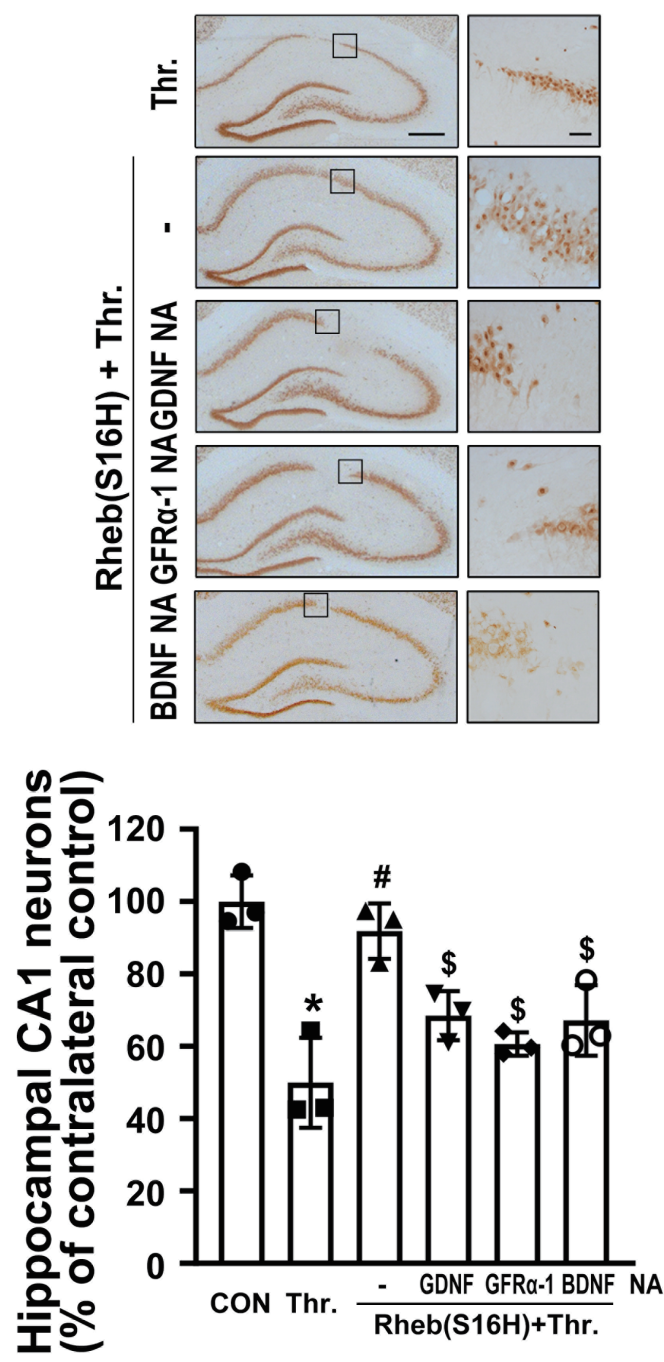

Fig. 5. Activation of the GDNF/GFR $\alpha$-1 signaling pathway contributes to Rheb(S16H)-induced neuroprotection in the hippocampus. Rats received an AAV injection, and then immunohistochemical staining for NeuN was performed to count the number of preserved neurons in the CA1 regions of the hippocampus at 7 days after treatment with thrombin (Thr; $20 \mathrm{U}$ ) and neutralizing antibodies (NA; $200 \mathrm{ng}$ ) against GDNF, GFRa-1, or BDNF, or thrombin alone (Fig. 1A). Right panels are magnified photomicrographs of the rectangular insets in the CA1 layer. Scale bars: left column, $500 \mu \mathrm{m}$; right column (insets), $100 \mu \mathrm{m}$. The number of NeuNpositive hippocampal neurons in each CA1 layer was quantitatively expressed as a percentage compared to that of the contralateral control. Differences among groups were evaluated by one-way analysis of variance and Tukey's post-hoc analysis; [ ${ }^{*} \mathrm{p}<0.001, \# \mathrm{p}<0.001$, and ${ }^{5} \mathrm{p}<0.05$ vs. CON, thrombin alone, $\mathrm{Rheb}(\mathrm{S} 16 \mathrm{H})+$ thrombin, respectively; $\mathrm{n}=3$ per group]

\section{DISCUSSION}

GDNF is an important neurotrophic factor that protects neurons in the brain, and the activation of the GDNF/GFR $\alpha$-1 signaling pathway modulates neuronal survival in various adult neurons $[1,10,12,20,21]$. Other reports show that the expression levels of
GDNF and GFR $\alpha-1$ are dysregulated in patients with neurodegenerative diseases such as Alzheimer's disease [3-5], and that the upregulation of GDNF by drug treatments or gene delivery could induce hippocampal neuroprotection $[22,23]$. Thus, the consistent upregulation of the GDNF-mediated signaling pathway can be considered an effective strategy against neurotoxicity and neurodegeneration in the adult brain.

GFRa-1 is a receptor specific for GDNF, and many of the cellular effects of its binding to GFR $\alpha$ - 1 are mediated by a cascade of intracellular signaling pathways, most notably that of Ret tyrosine kinase, which results in the downstream activation of the Akt prosurvival pathway that includes mTORC1 activation [10, 12, 24, 25]. Recently, Konishi et al. [4] reported that neurons lacking GFRa-1 did not respond to GDNF with neuronal survival, whereas production of GFR $\alpha-1$ in neurons with dysregulated GFR $\alpha$ - 1 restored the GDNF-mediated signaling pathway for neuronal survival, suggesting that the upregulation of GFRa-1 is also important for the induction of neuroprotective effects in the adult brain.

mTORC1, which can be stimulated by various neurotrophic factors, is a serine/threonine kinase that acts as a neuronal survival factor in the adult brain $[15,26]$. We previously reported that the sustained activation of mTORC1 by neuronal transduction with Rheb $(\mathrm{S} 16 \mathrm{H})$ could induce the production of neurotrophic factors such as BDNF and GDNF in dopaminergic neurons in the substantia nigra of adult brain in vivo [10], and that the increases in neurotrophic factors following Rheb(S16H) transduction contributed to neuroprotection in an animal model of Parkinson's disease [10].

Similar to the upregulation of BDNF in dopaminergic neurons in the substantia nigra [10], we recently reported that Rheb $(\mathrm{S} 16 \mathrm{H})$ transduction of hippocampal neurons could induce the production of neuronal BDNF through the activation of an mTORC1mediated signaling pathway in vivo, resulting in protection of hippocampal neurons from thrombin-induced neurotoxicity [14]. In the present study, we additionally found that increases in GDNF expression following Rheb(S16H) transduction were observed both in neurons and astrocytes in the hippocampus of rat brain (Fig. 2B), even though the transduction of Rheb(S16H) using AAV1 was mainly observed in neurons (Fig. 1B) [14]. These findings suggest that activation of the neurotrophic signaling pathway may occur through interactions between neurons and astrocytes in the Rheb(S16H)-transduced hippocampus.

In addition, GFRa-1 upregulation was also observed both in neurons and astrocytes in the Rheb(S16H)-transduced hippocampus (Fig. 3A and 3B), and GDNF treatment, which could stimulate the induction of GFR $\alpha-1$ in neurons [19], increased GFR $\alpha-1$ expression in astrocyte cultures (Fig. 4C). Consistent with our results, 


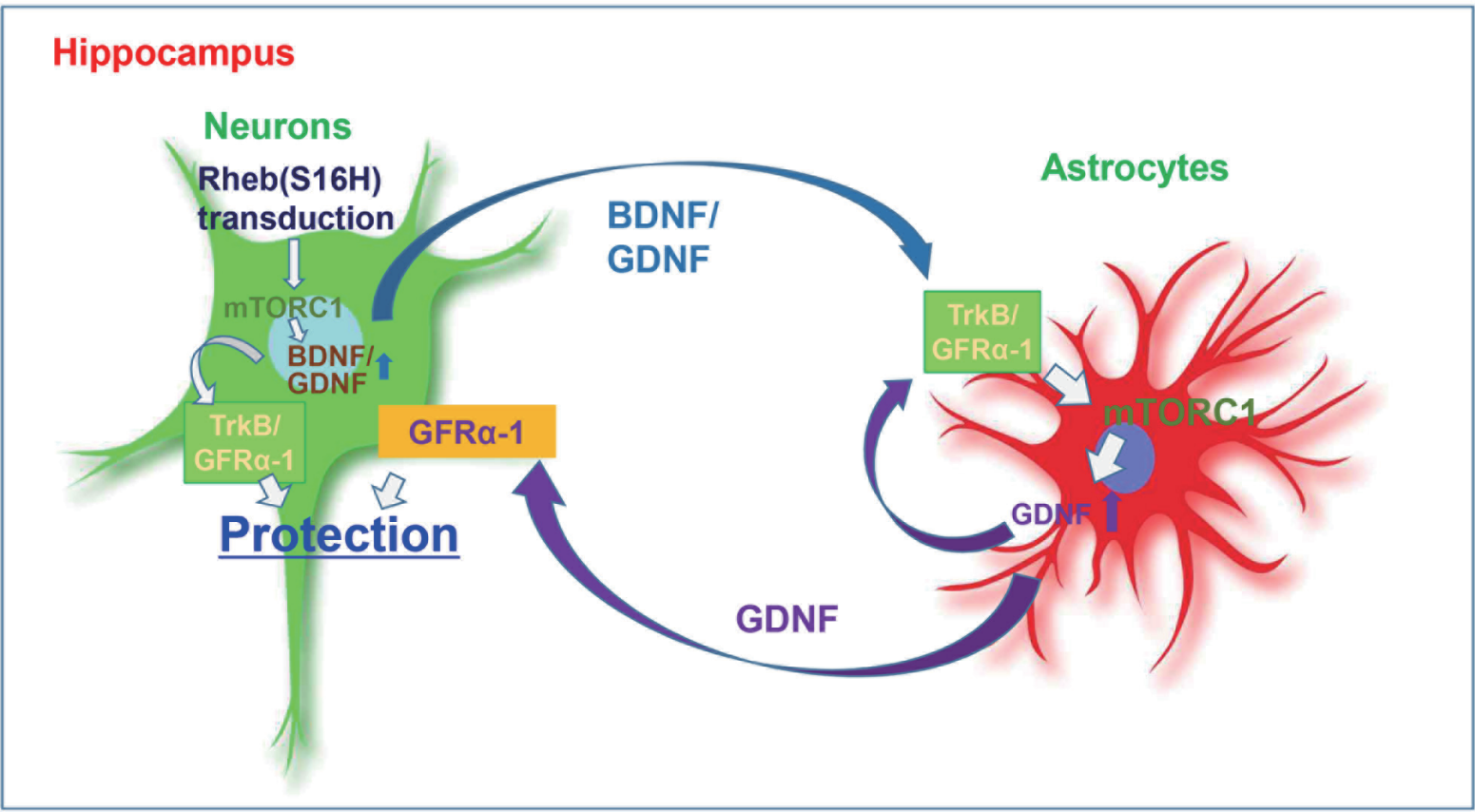

Fig. 6. Representative schematic of neurotrophic signaling pathway in the Rheb(S16H)-transduced hippocampus. Rheb(S16H) transduction of hippocampal neurons causes the induction of neurotrophic factors such as BDNF and GDNF. Increases in those factors stimulates astroglial activation, resulting in additional GDNF production. The induction of neurotrophic factors in both neurons and astrocytes contributes to neuroprotection in the adult hippocampus.

previous studies showed that GDNF could exert a trophic effect on astrocytes through GFRa-1 activation [27], and that GFRa-1 might be a mediator of autoregulation of GDNF $[28,29]$. BDNF could also act as a potent inducer for GFRa-1 expression in neurons [18]. However, our results show that neutralization of BDNF did not alter astrocytic GFRa-1 upregulation in the Rheb(S16H)expressed hippocampus (Fig. 4B), even though its neutralization decreased the expression of neuronal GFRa-1 (Fig. 4B). Moreover, treatment with BDNF did not increase GFR $\alpha-1$ expression in astrocyte cultures (Fig. 4D), suggesting that the upregulation of neuronal GDNF following Rheb(S16H) transduction might stimulate astrocytic GFRa-1 activation and upregulation in the hippocampus in vivo.

Although the induction of BDNF following Rheb(S16H) transduction may be not associated with astrocytic GFRa-1 upregulation, there are many reports showing that BDNF has the potential to induce trophic effects in astrocytes [30-33]. Furthermore, we recently reported that the increase in neuronal BDNF following Rheb(S16H) transduction could stimulate astroglial activation to produce ciliary neurotrophic factor, also contributing to neuroprotection as well as BDNF [34]. As demonstrated in Fig. 4A and 4B, the inhibition of GDNF and BDNF activity in the Rheb(S16H)treated hippocampus attenuated the upregulation of GDNF in both neurons and astrocytes. Collectively, our results suggest that the upregulation of astrocytic GFRa-1 following Rheb(S16H) transduction of hippocampal neurons may be mediated by the increase in GDNF expression, but not by BDNF, and that astrocytic GDNF upregulation through the activation of neurotrophic signaling pathways between neurons and astrocytes can contribute to protection of hippocampal neurons against neurotoxicity (Fig. 5).

In summary, we have identified additional mediators, GDNF and GFRa-1, that are associated with Rheb(S16H)-induced neuroprotection in the hippocampus in vivo. In addition to the production of neuronal BDNF following Rheb(S16H) transduction [14], the present study demonstrates that Rheb(S16H) transduction of hippocampal neurons stimulates the induction of GDNF and GFRa1 in neurons and astrocytes. Furthermore, our results show that the activation of the GDNF/GFRa-1 signaling pathway, an effect that can be indirectly stimulated by neuronal BDNF, occurs through the upregulation of those factors, and contributes to neuroprotection against thrombin-induced neurotoxicity in the hippocampus (Fig. 6).

\section{ACKNOWLEDGEMENTS}

This study was supported by grants from the National Research 
Foundation of Korea (NRF-2020R1A2C2007954), and the Korea Health Technology R\&D Project through the Korea Health Industry Development Institute (KHIDI), funded by the Ministry of Health and Welfare (HI14C1135), of the Korean government.

\section{CONFLICT OF INTEREST}

The authors declare no competing financial interests.

\section{AUTHOR CONTRIBUTIONS}

D.Y., M.T.J., H.J.K., and S.R.K. conceived and designed the experiments. D.Y., M.T.J., G.J.M., and S.R.K. conducted the experiments in vivo, analyzed the data, and generated the Figures. H.J.K. C.M.H., and S.L. conducted the experiments in vitro, and analyzed the data. M.S., and S.R.K. designed and provided the viral vectors. S.R.K. supervised the whole project and wrote the paper. All authors contributed to data analysis and the preparation of the manuscript.

\section{REFERENCES}

1. Paratcha G, Ledda F (2008) GDNF and GFRalpha: a versatile molecular complex for developing neurons. Trends Neurosci 31:384-391.

2. Ayanlaja AA, Zhang B, Ji G, Gao Y, Wang J, Kanwore K, Gao D (2018) The reversible effects of glial cell line-derived neurotrophic factor (GDNF) in the human brain. Semin Cancer Biol 53:212-222.

3. Airavaara M, Pletnikova O, Doyle ME, Zhang YE, Troncoso JC, Liu QR (2011) Identification of novel GDNF isoforms and cis-antisense GDNFOS gene and their regulation in human middle temporal gyrus of Alzheimer disease. J Biol Chem 286:45093-45102.

4. Konishi Y, Yang LB, He P, Lindholm K, Lu B, Li R, Shen Y (2014) Deficiency of GDNF receptor GFRal in Alzheimer's neurons results in neuronal death. J Neurosci 34:13127-13138.

5. Forlenza OV, Miranda AS, Guimar I, Talib LL, Diniz BS, Gattaz WF, Teixeira AL (2015) Decreased neurotrophic support is associated with cognitive decline in non-demented subjects. J Alzheimers Dis 46:423-429.

6. Lin LF, Doherty DH, Lile JD, Bektesh S, Collins F (1993) GDNF: a glial cell line-derived neurotrophic factor for midbrain dopaminergic neurons. Science 260:1130-1132.

7. Pascual A, Hidalgo-Figueroa M, Piruat JI, Pintado CO, Gómez-Díaz R, López-Barneo J (2008) Absolute requirement of GDNF for adult catecholaminergic neuron survival. Nat
Neurosci 11:755-761.

8. Maruyama W, Nitta A, Shamoto-Nagai M, Hirata Y, Akao Y, Yodim M, Furukawa S, Nabeshima T, Naoi M (2004) NPropargyl-1 (R)-aminoindan, rasagiline, increases glial cell line-derived neurotrophic factor (GDNF) in neuroblastoma SH-SY5Y cells through activation of NF-kappaB transcription factor. Neurochem Int 44:393-400.

9. Weinreb O, Amit T, Bar-Am O, Youdim MB (2007) Induction of neurotrophic factors GDNF and BDNF associated with the mechanism of neurorescue action of rasagiline and ladostigil: new insights and implications for therapy. Ann NY Acad Sci 1122:155-168.

10. Nam JH, Leem E, Jeon MT, Jeong KH, Park JW, Jung UJ, Kholodilov N, Burke RE, Jin BK, Kim SR (2015) Induction of GDNF and BDNF by hRheb(S16H) transduction of SNpc neurons: neuroprotective mechanisms of $\mathrm{hRheb}(\mathrm{S} 16 \mathrm{H})$ in a model of Parkinson's disease. Mol Neurobiol 51:487-499.

11. Aron L, Klein R (2011) Repairing the Parkinsonian brain with neurotrophic factors. Trends Neurosci 34:88-100.

12. Kholodilov N, Kim SR, Yarygina O, Kareva T, Cho JW, Baohan A, Burke RE (2011) Glial cell line-derived neurotrophic factor receptor- $\alpha 1$ expressed in striatum in trans regulates development and injury response of dopamine neurons of the substantia nigra. J Neurochem 116:486-498.

13. Choi SH, Lee DY, Kim SU, Jin BK (2005) Thrombin-induced oxidative stress contributes to the death of hippocampal neurons in vivo: role of microglial NADPH oxidase. J Neurosci 25:4082-4090.

14. Jeon MT, Nam JH, Shin WH, Leem E, Jeong KH, Jung UJ, Bae YS, Jin YH, Kholodilov N, Burke RE, Lee SG, Jin BK, Kim SR (2015) In vivo AAV 1 transduction with hRheb(S16H) protects hippocampal neurons by BDNF production. Mol Ther 23:445-455.

15. Kim SR, Chen X, Oo TF, Kareva T, Yarygina O, Wang C, During M, Kholodilov N, Burke RE (2011) Dopaminergic pathway reconstruction by Akt/Rheb-induced axon regeneration. Ann Neurol 70:110-120.

16. Paxinos G, Watson C (2007) The rat brain in stereotaxic coordinates. 6th ed. Academic Press, Amsterdam.

17. McCarthy KD, de Vellis J (1980) Preparation of separate astroglial and oligodendroglial cell cultures from rat cerebral tissue. J Cell Biol 85:890-902.

18. Honma M, Namikawa K, Mansur K, Iwata T, Mori N, Iizuka H, Kiyama H (2002) Developmental alteration of nerve injury induced glial cell line-derived neurotrophic factor (GDNF) receptor expression is crucial for the determination of injured motoneuron fate. J Neurochem 82:961-975. 
19. Bonafina A, Fontanet PA, Paratcha G, Ledda F (2018) GDNF/ GFRal complex abrogates self-renewing activity of cortical neural precursors inducing their differentiation. Stem Cell Reports 10:1000-1015.

20. Chen SS, Yang C, Hao F, Li C, Lu T, Zhao LR, Duan WM (2014) Intrastriatal GDNF gene transfer by inducible lentivirus vectors protects dopaminergic neurons in a rat model of parkinsonism. Exp Neurol 261:87-96.

21. Aly AE, Harmon BT, Padegimas L, Sesenoglu-Laird O, Cooper MJ, Waszczak BL (2019) Intranasal delivery of pGDNF DNA nanoparticles provides neuroprotection in the rat 6-hydroxydopamine model of Parkinson's disease. Mol Neurobiol 56:688-701.

22. Paterniti I, Cordaro M, Campolo M, Siracusa R, Cornelius C, Navarra M, Cuzzocrea S, Esposito E (2014) Neuroprotection by association of palmitoylethanolamide with luteolin in experimental Alzheimer's disease models: the control of neuroinflammation. CNS Neurol Disord Drug Targets 13:15301541.

23. Revilla S, Ursulet S, Álvarez-López MJ, Castro-Freire M, PerpiñáU, García-Mesa Y, Bortolozzi A, Giménez-Llort L, Kaliman P, Cristòfol R, Sarkis C, Sanfeliu C (2014) Lenti-GDNF gene therapy protects against Alzheimer's disease-like neuropathology in 3xTg-AD mice and MC65 cells. CNS Neurosci Ther 20:961-972.

24. Sariola H, Saarma M (2003) Novel functions and signalling pathways for GDNF. J Cell Sci 116(Pt 19):3855-3862.

25. Budni J, Bellettini-Santos T, Mina F, Garcez ML, Zugno AI (2015) The involvement of BDNF, NGF and GDNF in aging and Alzheimer's disease. Aging Dis 6:331-341.

26. Kim SR, Kareva T, Yarygina O, Kholodilov N, Burke RE (2012) AAV transduction of dopamine neurons with constitutively active Rheb protects from neurodegeneration and mediates axon regrowth. Mol Ther 20:275-286.

27. Bresjanac M, Antauer G (2000) Reactive astrocytes of the quinolinic acid-lesioned rat striatum express GFRalphal as well as GDNF in vivo. Exp Neurol 164:53-59.

28. He DY, Ron D (2006) Autoregulation of glial cell line-derived neurotrophic factor expression: implications for the longlasting actions of the anti-addiction drug, Ibogaine. FASEB J 20:2420-2422.

29. Barak S, Ahmadiantehrani S, Kharazia V, Ron D (2011) Positive autoregulation of GDNF levels in the ventral tegmental area mediates long-lasting inhibition of excessive alcohol consumption. Transl Psychiatry 1:e60.

30. Gaidin SG, Turovskaya MV, Gavrish MS, Babaev AA, Mal'tseva VN, Blinova EV, Turovsky EA (2020) The selective BDNF overexpression in neurons protects neuroglial networks against OGD and glutamate-induced excitotoxicity. Int J Neurosci 130:363-383.

31. Lin CH, Wang CH, Hsu SL, Liao LY, Lin TA, Hsueh CM (2016) Molecular mechanisms responsible for neuron-derived conditioned medium (NCM)-mediated protection of ischemic brain. PLoS One 11:e0146692.

32. Harada C, Guo X, Namekata K, Kimura A, Nakamura K, Tanaka K, Parada LF, Harada T (2011) Glia- and neuron-specific functions of TrkB signalling during retinal degeneration and regeneration. Nat Commun 2:189.

33. Harada C, Harada T, Quah HM, Maekawa F, Yoshida K, Ohno S, Wada K, Parada LF, Tanaka K (2003) Potential role of glial cell line-derived neurotrophic factor receptors in Müller glial cells during light-induced retinal degeneration. Neuroscience 122:229-235.

34. Jeon MT, Moon GJ, Kim S, Choi M, Oh YS, Kim DW, Kim HJ, Lee KJ, Choe Y, Ha CM, Jang IS, Nakamura M, McLean C, Chung WS, Shin WH, Lee SG, Kim SR (2020) Neurotrophic interactions between neurons and astrocytes following AAV1-Rheb $(\mathrm{S} 16 \mathrm{H})$ transduction in the hippocampus in vivo. Br J Pharmacol 177:668-686. 